\title{
Consumo de tabaco y práctica de actividad física en una población melillense
}

\section{Tobacco use and practice of physical activity in a melillense population}

\author{
Silvia San Román-Mata ${ }^{1}$, Andrés Fernández-Revelles ${ }^{2}$, Pedro Valdivia-del Moral ${ }^{3}$, Asunción Martínez-Martínez ${ }^{4 *}$ \\ y Manuel Castro-Sánchez ${ }^{5}$
}

\author{
${ }^{1}$ Departamento de Enfermería, Facultad de Ciencias de la Salud de Melilla, Universidad de Granada (España). \\ ${ }^{2}$ Departamento de Educación Física y Deportiva, Facultad de Ciencias del Deporte, Universidad de Granada (España). \\ ${ }^{3}$ Departamento de Didáctica de la Expresión Musical, Plástica y Corporal, Facultad de Ciencias de la Educación, Universidad de Granada (España). \\ ${ }^{4}$ Departamento de Métodos de Investigación y Diagnóstico en Educación, Facultad de Ciencias de la Educación, Universidad de Granada (España). \\ ${ }^{5}$ Castro-Sánchez, Manuel: Departamento de Didáctica de la Expresión Musical, Plástica y Corporal, Facultad de Ciencias Educación y del Deporte de Melilla, \\ Universidad de Granada (España).
}

Resumen: Introducción: El consumo de tabaco es considerado factor de riesgo ante el desarrollo de múltiples patologías a nivel pulmonar y cardio-vascular, entre otras. En este sentido, la práctica de actividad física es considerada un factor de protección. Objetivo: Describir las variables socio-demográficas y de calidad de vida en cuanto al consumo de tabaco y la práctica de actividad física en una población Melillense. Material y Método: Trabajo de investigación con diseño cuantitativo, de carácter descriptivo y corte transversal de una muestra de residentes en Melilla, acerca del consumo de tabaco y la práctica de actividad física. Resultados: Más de tres cuartas partes de los participantes manifestaron no fumar tabaco y tres de cada diez sujetos no realizaba ningún tipo de actividad física.

Conclusiones: Hay que seguir trabajando por afianzar conductas saludables en la población, en relación al consumo de sustancias nocivas y la práctica de actividad física.

Palabras clave: consumo, tabaco, actividad física, deporte
Abstract: Introduction: Tobacco use is considered a risk factor in the development of multiple pathologies at the pulmonary and cardio-vascular levels, among others. In this sense, the practice of physical activity is considered a factor of protection. Objective: To describe the socio-demographic variables and quality of life in terms of tobacco use and the practice of physical activity in a Melillense population.

Material and Method: Research work with quantitative design, descriptive and cross section of a sample of residents in Melilla, about tobacco use and the practice of physical activity. Results: More than three-quarters of the participants said no smoking and three out of ten subjects did not perform any physical activity. Conclusions: We must continue working to strengthen healthy behaviors in the population, in relation to the consumption of harmful substances and the practice of physical activity.

Keywords: consumption, tobacco, physical activity, sport

\section{Introducción}

El consumo de tabaco, es un hábito insano que se encuentra aceptado en la sociedad actual. En concreto en nuestro país, es considerado una droga legal accesible a la población, compuesta por nicotina y alquitrán, que puede ser comprada en multitud de establecimientos: estancos, aeropuertos, quioscos, restaurantes, gasolineras...y aunque se encuentra restringida su venta a los menores de 18 años, la de edad de inicio en su consumo en muchos de los casos, se encuentra enmarcada en el periodo de la adolescencia, comenzando en las etapas académicas de educación secundaria y universitaria (Castro-Sánchez et al., 2017; Espinosa-Herrera y CastellanosObregón, 2017), lo que se demuestra su fácil accesibilidad.

Del mismo modo, el Ministerio de Sanidad, Servicios Sociales e Igualdad (2016), en su encuesta estatal dirigida a alumnos de secundaria, constata la presencia del tabaco a estas edades ya que un $44 \%$ de los estudiantes manifestó haber probado el tabaco. Por tanto, el consumo de tabaco es un hecho considerado problema de salud pública y de interés social (Barragán et al., 2016).

Dirección para correspondencia [Correspondence address]: Asunción Martínez-Martínez. E-mail: asuncionmm@ugr.es
Durante las últimas décadas, las investigaciones han constatado la influencia del tabaco en la salud, encontrando efectos perjudiciales de su uso a corto plazo que pueden degenerar en gripe, mal aliento o resfriados, entre otros. Pero a largo plazo, su consumo puede ocasionar e incidir en el desarrollo y evolución de otras patologías de mayor gravedad tales como la enfermedad pulmonar obstructiva crónica, el cáncer de pulmón o de boca, entre otras muchas (Chacón-Cuberos et al., 2016; Valdés-Iglesia, Vento-Lezcano, Hernández-Martínez, Álvarez-Gómez y Díaz-Pita, 2018).

Por lo que hay que prestar especial atención al colectivo adolescente, donde los cambios fisiológicos y de relación social son múltiples, las conductas impulsivas forman parte característica de esta etapa y en la que el desarrollo de la personalidad está en auge. En este sentido, cabe la posibilidad de adquirir e instaurar conductas insanas, manifestándose en la edad adulta lo que incide finalmente en la salud de la población. De manera que la intervención y los programas de educación para la salud, son de primera necesidad (San RománMata, 2018), para poder prevenir conductas disruptivas y al mismo tiempo fomentar hábitos y estilos saludables de vida.

Por otro lado, la Organización Mundial de la Salud junto 
con multitud de autores afirman la cantidad de beneficios que aporta la práctica de actividad física al organismo y a la salud mental de individuo, y por ende a la población y comunidad (Alcaraz, Pons, Angulo-Brunet y Cruz, 2017; Guillén-Pereira, Bueno-Fernández, Gutiérrez-Cruz y Guerra-Santisteban, 2018). De forma que la práctica de actividad física regular incorporada en la vida diaria, como hábito y estilo de vida saludable, se considera un factor de protección ante patologías, así como de conductas o hábitos insanos como puede ser el consumo de tabaco (Espejo et al., 2017).

Así mismo, con la práctica de ejercicio físico se desarrollan diversos aspectos, se aumenta la musculatura y la capacidad pulmonar, relacionándose igualmente con la autoimagen, mejorando el autoconcepto y autoestima. Del mismo modo, autores como García-González y Froment, (2018) manifiestan que su práctica fomenta el trabajo en equipo y facilita las relaciones sociales, así como el rendimiento académico (Martínez y González, 2017).

Pero a pesar de la información existente y cercana al día de hoy, en todos los medios de comunicación que nos rodean acerca de la práctica de actividad física y sobre el consumo de tabaco, la realidad es que parece no ser totalmente en la actualidad, especialmente en la adolescencia, ya que se observa una disminución de la práctica de actividad física, (BeltránCarrillo et al., 2017; Revuelta, Infante y Axpe, 2017), además del auge de conductas nocivas para la salud (San Román-Mata, 2019). Situación preocupante a nivel nacional e internacional (Zurita-Ortega et al., 2018), y de predisposición variable, tal y como afirma Molero-Jurado, Pérez-Fuentes, GázquezLinares y Barragán-Martin (2017).

Así pues, nuestro estudio pretende dar respuesta a los parámetros seleccionados en relación a las variables sociodemográficas, el consumo de tabaco y la práctica de actividad física. Exponiéndose como objetivo general de esta investigación describir las variables sociodemográficas y de calidad de vida en cuanto al consumo de tabaco y la actividad física en población Melillense. De este objetivo general, se desglosan y se proponen los siguientes objetivos específicos:

- Dictaminar las variables sociodemográficas de los participantes.

- Detallar el consumo de tabaco y la práctica de actividad física de la población de estudio.

- Mostrar las relaciones existentes entre las variables relacionadas con la calidad de vida sobre el consumo de tabaco y la práctica de actividad física según el género.

\section{Material y Métodos}

\section{Diseño y participantes}

En la presente investigación se utilizó un diseño cuantitativo de carácter descriptivo y corte transversal, llevándose a cabo un estudio relacional entre las variables analizadas. En este estudio han participado un total de 1.073 personas $(36,5 \%$ mujeres; $63,5 \%$ hombres) con edades comprendidas entre 18 y 65 años $(M=35,7 ; \mathrm{DT}=12,7)$ de nacionalidad espańola, residentes en la Ciudad Autónoma de Melilla, que han colaborado libremente en la investigación. La selección de la muestra se realizó mediante un muestreo de conveniencia.

\section{Variables e instrumentos}

La investigación tomó como referencia las siguientes variables e instrumentos:

Cuestionario de elaboración propia para el registro de las variables sociodemográficas: sexo, edad, domicilio de residencia, estado académico o laboral y nivel de estudios. Así como frecuencia de la práctica de actividad física semanalmente y de qué tipo (individual o colectivo)

Cuestionario FTND: Escala de consumo de tabaco de Heatherton, Kozlowski, Frecker y Fagerström (1991), y traducido al castellano por Villareal- González (2009). Compuesto por 6 preguntas, cuatro de ellas de carácter dicotómico y dos con escala likert de cuatro opciones. Estableciéndose finalmente cuatro categorías: no fumador, dependencia baja, moderada y alta.

\section{Procedimiento}

Tras contactar con los organismos pertinentes para la solicitud de permisos, se llevó a cabo el trabajo de campo, donde la recogida de datos, a pesar de poder realizarse on line mediante la plataforma de Google Drive, se pasó el cuestionario de manera presencial en diferentes aulas de la universidad y las empresas colaboradoras. Así mismo, los participantes lo rellenaron de forma voluntaria y anónima siguiendo los principios éticos y de confidencialidad de la Declaración de Helsinki (modificación del 2008), en cuanto a proyectos de investigación se refiere.

\section{Análisis de los resultados}

Para el análisis estadístico se utilizó el paquete estadístico SPSS 22.0., que mediante la utilización de frecuencias y medias se calcularon los descriptivos básicos, empleando las tablas de contingencia para la realización del estudio relacional. 


\section{Resultados}

Tomando como referencia los análisis descriptivos, concernientes a los 1073 participantes de este estudio, se evidenció que $681(63,5 \%)$ eran varones y 392 mujeres $(36,5 \%)$ con un rango de edad de 18 a 65 ańos $(M=35,7 ;$ D.T=12,7) siendo en mayor medida el hogar familiar, el lugar de residencia de los mismos, y observándose un mayor porcentaje de personas con ocupación laboral (66,5\%) frente a estudiantes (31,5\%), tal y como puede apreciarse en la siguiente tabla:
Tabla 1. Distribución de variables sociodemográficas.

\begin{tabular}{cc}
\hline \multicolumn{2}{c}{ Género } \\
\hline Masculino & $63,5 \%(\mathrm{n}=681)$ \\
Femenino & $36,5 \%(\mathrm{n}=392)$ \\
\hline \multicolumn{2}{c}{ Residencia Habitual } \\
\hline Domicilio Familiar & $81,5 \%(\mathrm{n}=875)$ \\
Piso Compartido & $15,7 \%(\mathrm{n}=168)$ \\
Residencia universitaria & $2,0 \%(\mathrm{n}=21)$ \\
Residencia militar & $0,4 \%(\mathrm{n}=4)$ \\
Hotel & $0,5 \%(\mathrm{n}=5)$ \\
\hline \multicolumn{2}{c}{ Situación Laboral } \\
\hline Estudias & $31,5 \%(\mathrm{n}=338)$ \\
Trabajas & $66,5 \%(\mathrm{n}=714)$ \\
\hline
\end{tabular}

Igualmente, un $10 \%$ de los participantes presentaban estudios de primaria o secundaria, casi un 20\% disponían de estudios de bachillerato, el 16,4\% eran técnicos especialistas y más de la mitad de la población presentaban estudios superiores, como se muestra a continuación:

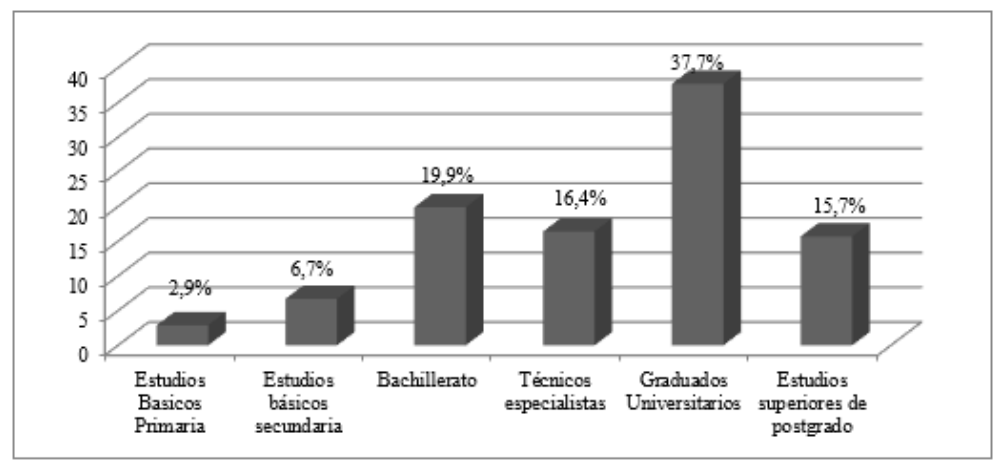

Figura 1. Distribución según nivel de estudios.

En relación al consumo de tabaco, se puede observar que más de tres cuartas partes de los participantes no fuman $(77,7 \%) \mathrm{y}$ que un 2,6\% de fumadores se presentan con una alta dependencia a esta sustancia (Figura 2).

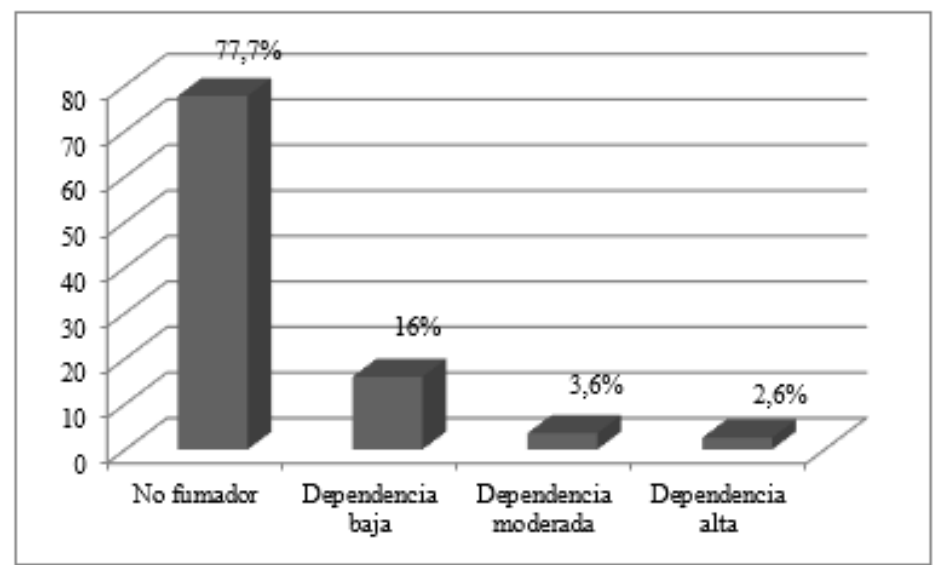

Figura 2. Distribución del consumo de tabaco. 
Además, no se encuentran diferencias estadísticamente significativas en relación al sexo $(p=0,199)$ en este sentido, como se muestra en la tabla 2.

Tabla 2. Distribución del consumo de tabaco según género $(\mathrm{p}=0,199)$.

\begin{tabular}{ccccccc}
\hline Género & Consumo de tabaco & No fumador & Dependencia baja & Dependencia moderada & Dependencia alta & Total \\
\hline \multirow{3}{*}{ Hombre } & Recuento & 537 & 109 & 22 & 13 & 681 \\
& \% Género & $78,9 \%$ & $16,0 \%$ & $3,2 \%$ & $1,9 \%$ & $100 \%$ \\
& \% Consumo & $64,4 \%$ & $63,4 \%$ & $56,4 \%$ & $46,4 \%$ & $63,5 \%$ \\
\hline \multirow{3}{*}{ Mujer } & Recuento & 297 & 63 & 17 & 15 & 392 \\
& \% Género & $75,8 \%$ & $16,1 \%$ & $4,3 \%$ & $3,8 \%$ & $100 \%$ \\
& \% Consumo & $35,6 \%$ & $36,6 \%$ & $33,6 \%$ & 28 & 36,5 \\
\hline \multirow{3}{*}{ Total } & Recuento & 834 & 172 & $3,6 \%$ & $2,6 \%$ & 1073 \\
& \% Género & $77,7 \%$ & $16,0 \%$ & $100 \%$ & $100 \%$ & $100 \%$ \\
\hline
\end{tabular}

Por otro lado, y en relación a la práctica de actividad física, nuestros hallazgos indican que un $31,7 \%$ de los sujetos no realiza ninguna actividad, frente al $68,3 \%$ que si la realiza (Figura 3).

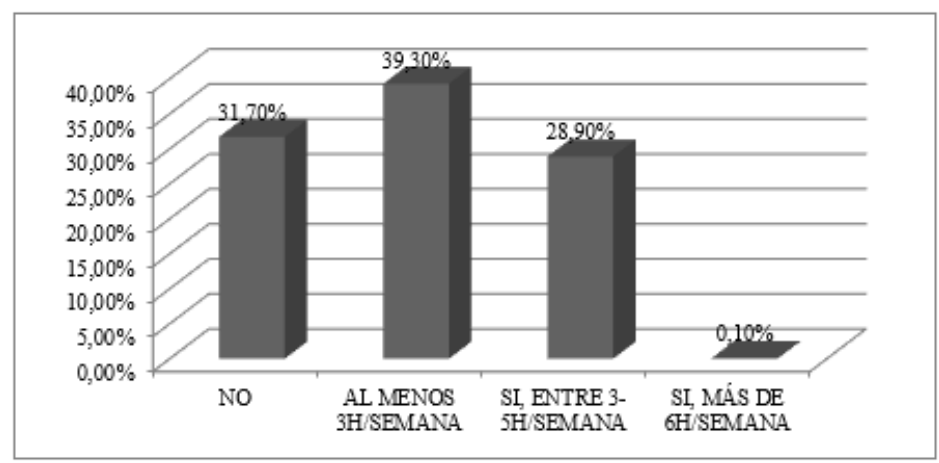

Figura 3. Distribución frecuencia de actividad física.

En este sentido y al relacionar la frecuencia de la actividad física según el género, se constatan en nuestros resultados, diferencias estadísticamente significativas al respecto $(p=0,000)$. De forma que el porcentaje de varones $(36,7 \%)$ frente a mujeres $(26,5 \%)$ que no realizan actividad física es mayor, $y$ mientras el varón obtiene mayores porcentajes en realizar deporte al menos 3 horas a la semana, la mujer se presenta más constante en la práctica de actividad física semanal con más de 5 horas semanales. A continuación, se muestran los datos desglosados en la siguiente tabla:

Tabla 3. Distribución de la frecuencia de actividad física según género $(\mathrm{p}=0,000)$.

\begin{tabular}{lllllll}
\hline Género & Actividad Física & No & Si, al menos 3h/semana & Si, entre 3-5h/semana & Si, mas de 6h/semana & Total \\
\hline \multirow{2}{*}{ Hombre } & Recuento & 250 & 295 & 135 & 1 & 681 \\
& \% Género & $36,7 \%$ & $43,3 \%$ & $19,8 \%$ & $0,1 \%$ & $100 \%$ \\
& \% Actividad Física & $73,5 \%$ & $70,2 \%$ & $43,5 \%$ & $33,3 \%$ & $63,5 \%$ \\
\hline \multirow{2}{*}{ Mujer } & Recuento & 90 & 125 & 175 & 2 & 392 \\
& \% Género & 23,08 & $31,9 \%$ & $44,6 \%$ & 0,5 & $100 \%$ \\
& \% Actividad Física & $26,5 \%$ & $29,8 \%$ & $56,5 \%$ & $66,7 \%$ & $36,5 \%$ \\
\multirow{2}{*}{ Total } & Recuento & 340 & 420 & 310 & 3 & 1073 \\
& \% Género & 31,7 & $39,1 \%$ & $28,9 \%$ & 0,3 & $100 \%$ \\
\hline
\end{tabular}




\section{Discusión}

El presente estudio indica un consumo inferior de tabaco con respecto a los llevados a cabo por García-Díaz, FernándezFeito, Arias y Lana (2015), resultando ser más alentadores. Así mismo, nuestros hallazgos coinciden con resultados de otros autores tales como Fuentes, Alarcón, García y Gracia (2015), y Giménez-García, Ruiz-Palomino, Gil-Llario, Ballester-Arnal y Castro-Calvo (2016), en cuanto a que no existen diferencias estadísticamente significativas al relacionar el sexo con el consumo de tabaco. Este hecho, nos sugiere un cambio en el hábito tabáquico de la mujer, que años atrás mostraba puntuaciones bastante inferiores a las obtenidas por los hombres al día de hoy. Es evidente que el cambio familiar, laboral, social y cultural que se ha producido en la mujer en las últimas décadas, ha influido en sus conductas y estilos de vida.

Es por ello que Calvete y Estévez (2009) y Zaranza-Monteiro et al. (2018), obtienen mayores puntuaciones por parte de las chicas en el hábito tabáquico con respecto a los chicos. De hecho, Rial et al. (2018) aconsejan prestar mayor atención al colectivo femenino por el auge producido durante los últimos ańos.

En relación a la práctica de educación física, nuestros resultados confirman un descenso de la práctica de actividad física de la población en general, al igual que lo exponen Castro-Sanchez, Linares-Manrique, San Román-Mata y PérezCortés (2017) al constatar que alrededor de4 de cada 10 de sus participantes no realizaba ejercicio físico. En este sentido, López-Sánchez, González-Villora y Diaz-Suarez (2016) y López-Sánchez, Ahmed y Díaz-Suarez (2017), afirman un descenso de la actividad física de especial interés en la última fase de la adolescencia y durante la primera etapa adulta.

Así mismo, Práxedes, Sevil, Moreno, del Villar y GarcíaGonzález (2016), exponen la etapa universitaria como período crítico, en donde más de la mitad de los universitarios estudiados no llegan a desarrollar el mínimo de actividad física diaria recomendada, siendo los varones los que mayor grado de cumplimiento presentan y considerando esta etapa como factor de predicción en la vida adulta. Sin embargo, en nuestros resultados, el sexo femenino es el que presenta mayor práctica de actividad física, lo que nos sugiere ser un fenómeno de carácter variable y que necesita ser estudiado con mayor profundidad.

Muchas podrían ser las explicaciones de la inactividad física, desde la simple vagancia o desgana de la población, el can- sancio y la falta de tiempo al día para desarrollar actividades físicas y deportivas, el uso desmesurado de nuevas tecnologías (ordenadores, videojuegos, telefonía móvil, televisión...) o la elección de otras actividades de ocio y tiempo libre de carácter sedentario, entre otras.

En este sentido, Martín-Rodriguez et al. (2018) analizan la relación existente entre las diferencias sociológicas de los individuos (situación demográfica, clase social, nivel de estudios) y las demandas de actividad física, de forma que, en sus resultados, se puede observar la influencia de dichas variables en la práctica deportiva, resaltando la clase social como un parámetro de interés de estudio, siendo las personas de clase social baja las que menos demandan realizar deporte.

\section{Conclusiones}

Como principales conclusiones de la investigación llevada a cabo podemos decir:

- En este trabajo se constató que 6 de cada diez participantes eran varones y que ocho de cada diez residían en el hogar familiar. Así mismo más de la mitad disponían de estudios universitarios.

- En referencia al consumo de tabaco, se evidenció que más de tres cuartas partes de la población era no fumadora.

- No se evidenció diferencias estadísticamente significativas en relación al consumo de tabaco según el género.

- Se confirmó que 3 de cada diez participantes no realizaba actividad física.

- Se constató existir diferencias estadísticamente significativas en cuanto a la práctica de actividad física y género, resultando ser el sexo femenino el que mayor grado de cumplimiento y constancia presenta.

Teniendo en cuenta los hallazgos obtenidos en nuestra investigación, se proponen como líneas de investigación futuras la implantación de programas de prevención y educación para la salud acerca del consumo de sustancias nocivas, seguimiento y evaluación de los mismos, prestando especial atención al colectivo femenino. Así como programas de actividad física instaurados desde edad infantil y actividades deportivas inclusivas y comunitarias de fácil acceso para toda la población. Igualmente se sugieren la ampliación y desarrollo de investigaciones y estudios acerca de la práctica de actividad física, que incluyan nuevos parámetros y variables de estudio relacionados.

\section{Referencias Bibliográficas}

1. Alcaraz, S., Pons, J., Angulo-Brunet, A., y Cruz, J. (2017). Intervenciones para la promoción de actividad física basadas en la teoría de la autodeterminación: Una revisión narrativa (2011-2016). Revista psicología del deporte, 26(3), 116-123.
2. Barragán, A.B., Martos, A., Simón, M., Pérez-Fuentes, M., Molero, M., y Gázquez, J.J. (2016). Consumo de tabaco y alcohol en adolescentes y relación con la familia. European Journal of Child Development, Education and Psychopathology, 4(1), 49-61. 
3. Beltrán-Carrillo, V., Sierra, C., Jiménez-Loaisa, A., González-Cutre, D., Martínez-Galindo, C., y Cervelló, E. (2017). Diferencias según género en el tiempo empleado por adolescentes en actividad sedentaria y actividad física en diferentes segmentos horarios del día. Retos, 31, 3-7.

4. Calvete, E., y Estévez, A. (2009). Consumo de drogas en adolescentes: El papel del estrés, la impulsividad y los esquemas relacionados con la falta de límites. Adicciones, 21(1), 49-56.

5. Castro-Sánchez, M., Linares-Manrique, M., San-Román-Mata, S., y Cortés-Pérez, A. J. (2017). Análisis de los comportamientos sedentarios, práctica de actividad física y uso de videojuegos en adolescentes. Sportis. Scientific Journal of School Sport, Physical Education and Psychomotricity, 3(2), 241-255.

6. Castro-Sánchez, M., Zurita-Ortega, F., Chacón-Cuberos, R., EspejoGarcés, T., Martínez-Martínez, A. y Pérez-Cortés, A. J. (2017). Sustancias nocivas y actividad física en adolescentes. Sportis, 3(2), 223-240.

7. Chacón-Cuberos, R., Castro-Sánchez, M., Caracuel-Cáliz, R., PadialRuz, R., Collado-Fernández, D. y Zurita-Ortega, F. (2016). Perfiles de consumo de alcohol y tabaco en adolescentes andaluces de primer ciclo de educación secundaria. Health and Adicctions, 16(2), 93-104.

8. Espejo-Garcés, T., Martínez, A., Chacón, R., Zurita, F., Castro, M., y Chacón, J. (2017). Consumo de alcohol y actividad física en adolescentes de entorno rural. Health and Addictions/Salud y Drogas, 17(1), 97-105.

9. Espinosa-Herrera, G., y Castellanos-Obregón, J.M. (2017). Procesos de estructuración de prácticas trasgresoras asociadas al consumo de sustancias psicoactivas en universitarios. Revista Latinoamericana de Ciencias Sociales, Niñez y Juventud, 16(2), 777-795.

10. Fuentes, M., Alarcón, A., García, F., y Gracia, E. (2015). Consumo de alcohol, tabaco, cannabis y otras drogas en la adolescencia: efectos de la familia y peligro del barrio. Anales de psicología, 31(3), 1000-1007.

11. García-Díaz, V., Fernández-Feito, A., Arias, L., y Lana, A. (2015). Consumo de tabaco y alcohol según la jornada laboral en Espańa. Gaceta Sanitaria, 29(5), 364-369.

12. García-González, A. J. y Froment, F. (2018). Beneficios de la actividad física sobre la autoestima y la calidad de vida de personas mayores. Retos, 33(33), 3-9.

13. Giménez-García, C., Ruiz-Palomino, E., Gil-Llario, M.D., BallesterArnal, R., y Castro-Calvo, J. (2016). Una perspectiva de género en el estudio de conductas de riesgo de los adolescentes. Infad Revista de Psicología, 1(2), 189-198.

14. Guillén-Pereira, L., Bueno-Fernández, E., Gutiérrez-Cruz, M. y Guerra-Santiesteban, J. R. (2018). Programa de actividad física y su incidencia en la depresión y bienestar subjetivo de adultos mayores. Retos, 33(33), 14-19.

15. López-Sánchez, G.F., Ahmed, D., y Díaz-Suárez, A. (2017). Level of habitual physical activity among 13 years-old adolescents from Spain and India. A cross-cultural study. SPORT TK: Revista EuroAmericana de Ciencias del Deporte, 6(1), 67-74.

16. López-Sánchez, G.F., González-Víllora, S., y Díaz-Suárez, A. (2016) Level of habitual physical activity in children and adolescents from the Region of Murcia. SpringerPlus, 5(386), 1-6.

17. Martín-Rodrígez, M., Espada-Mateo, M., Moscoso-Sánchez, D., Jiménez-Beatty, J.E., Santacruz-Lozano, J.A., y Jiménez-Díaz, V. (2018). La práctica de actividad física y deporte: una demanda sociológicamente construida. Revista de Humanidades, 34, 87-105.

18. Martínez, F. D., y González, J. (2017). Autoconcepto, práctica de actividad física y respuesta social en adolescentes: Relaciones con el rendimiento académico. Revista Iberoamericana de Educación, 73(1), 5, 87108.

19. Molero-Jurado, M.M., Pérez-Fuentes, M.C., Gázquez-Linares, J.J., y Barragán-Martin, A.B. (2017). Análisis y perfiles del consumo de drogas en adolescentes: percepción del apoyo familiar y valoración de consecuencias. Mediagraphic, 24(2), 56-61.

20. Práxedes, A., Sevil, J., Moreno, A., del Villar, F., y García-González, L. (2016). Niveles de actividad física en estudiantes universitarios: diferencias en función del género, edad y los estados de cambio. Revista Iberoamericana de Psicología del Ejercicio y el Deporte, 11(1), 123-132.

21. Revuelta, L., Infante, G., y Axpe, I. (2017). Contexto familiar e implicación en el ámbito físico-deportivo durante la adolescencia. International Journal of Developmental and Educational Psychology. Revista INFAD de Psicología., 6(1), 311-320.

22. Rial, A., Burkhardt, G., Isorna, M., Barreiro, C., Varela, J., y Golpe, S. (2018). Consumo de cannabis entre adolescentes: patrón de riesgos, implicaciones y posibles variables explicativas. Adicciones, 20(10), 1-14.

23. San Román-Mata, S. (2018). Análisis del perfil del estudiante universitario en relación a hábitos y estilos de vida saludables, motivaciones y expectativas socioeducaticas. Tesis doctoral: Universidad de Granada.

24. San Román-Mata, S. (2019). Consumo de tabaco y hachís. Diferencias de género. ESPHA, Education, Sport, Health and Physical Activity, 3(1), 107- 119 .

25. Valdés-Iglesia, A., Vento-Lezcano, C., Hernández-Martínez, D., Álvarez-Gómez, A. y Díaz-Pita, G. (2018). Drogas, un problema de salud mundial. Revista Universidad Médica Pinareña, 14(2), 168-183.

26. Zaranza-Monteiro, L., Ramírez-Varela, A., Rodriguez-Alves, L., Lopes, G., Júnior. M.A., y Beltrame, L. (2018). Prevalence of and factors associated with alcohol and tobacco consumption among physical education undergraduates. Journal physicology education, 29, 1-10.

27. Zurita-Ortega, F., Salvador-Pérez, F., Knox, E., Gámiz-Sánchez, V., Chacón-Cuberos, R., Rodríguez-Fernández, S. y Muros, J.J. (2018). Physical activity and health-related quality of life in schoolchildren: structural equations analysis. Anales de psicología, 34(2), 385-390. 